\title{
Pharmacokinetics of 17-hydroxyprogesterone caproate in multifetal gestation
}

\author{
Steve N. Caritis, $\mathrm{MD}^{1}$, Shringi Sharma, $\mathrm{PhD}^{2}$, Raman Venkataramanan, $\mathrm{PhD}^{2}$, Dwight J. \\ Rouse, $\mathrm{MD}^{3}$, Alan M. Peaceman, $\mathrm{MD}^{4}$, Anthony Sciscione, $\mathrm{DO}^{5}$, Catherine Y. Spong, $\mathbf{M D}^{6}$, \\ Michael W. Varner, MD ${ }^{7}$, Fergal D. Malone, MD $^{8}$, Jay D. lams, MD ${ }^{9}$, Brian M. Mercer, MD ${ }^{10}$, \\ John M Thorp Jr, MD ${ }^{11}$, Yoram Sorokin, MD ${ }^{12}$, Marshall Carpenter, MD ${ }^{13}$, Julie Lo, MD ${ }^{14}$, \\ Susan Ramin, MD ${ }^{15}$, and Margaret Harper, MD, MS ${ }^{16}$ for the Eunice Kennedy Shriver \\ National Institute of Child Health and Human Development Maternal-Fetal Medicine Units \\ Network \\ ${ }^{1}$ Departments of Obstetrics, Gynecology and Reproductive Sciences, Pittsburgh, PA \\ ${ }^{2}$ Department of Pathology University of Pittsburgh School of Medicine and the Department of \\ Pharmaceutical Sciences School of Pharmacy, Pittsburgh, PA \\ ${ }^{3}$ Departments of Obstetrics and Gynecology of University of Alabama at Birmingham, \\ Birmingham, AL \\ ${ }^{4}$ Northwestern University, Chicago, IL \\ ${ }^{5}$ Drexel University, Philadelphia, PA \\ ${ }^{6}$ Eunice Kennedy Shriver National Institute of Child Health and Human Development, Bethesda, \\ $\mathrm{MD}$ \\ ${ }^{7}$ University of Utah, Salt Lake City, UT \\ ${ }^{8}$ Columbia University, New York, NY
}

\author{
(C) 2011 Mosby, Inc. All rights reserved. \\ Publisher's Disclaimer: This is a PDF file of an unedited manuscript that has been accepted for publication. As a service to our \\ customers we are providing this early version of the manuscript. The manuscript will undergo copyediting, typesetting, and review of \\ the resulting proof before it is published in its final citable form. Please note that during the production process errors may be \\ discovered which could affect the content, and all legal disclaimers that apply to the journal pertain. \\ In addition to the authors, other members of the Eunice Kennedy Shriver National Institute of Child Health and Human Development \\ Maternal-Fetal Medicine Units Network are as follows: \\ University of Pittsburgh - S. Zhang, M. Cotroneo, H. Simhan \\ University of Alabama at Birmingham - W. Andrews, J. Sheppard, N. Northen, J Tillinghast \\ Northwestern University - M. Dinsmoor (Evanston Hospital), G. Mallet, P. Simon, M. Huntley,M. Ramos \\ Drexel University - M. Hoffman, S. Wilson, C. Tocci, M. Lake, M. Talucci \\ University of Utah - K. Anderson, F. Porter (LDS Hospital), A. Guzman (McKay-Dee Hospital Center), K. Jolley (Utah Valley \\ Regional Medical Center), S. Quinn (LDS Hospital) \\ Columbia University - R. Berkowitz, S. South, L. Paley, S. Bousleiman, V. Carmona, R. Wapner \\ The Ohio State University - F. Johnson, C. Latimer \\ Case Western University - C. Milluzzi, C. Heggie, H. Ehrenberg, B. Stetzer, A. Merlino \\ University of North Carolina at Chapel Hill-K. Boggess, K. Dorman, S. Timlin \\ Wayne State University - G. Norman, C. Sudz, S. Blackwell \\ Brown University - D. Allard \\ University of Texas Southwestern Medical Center, Dallas - K. Leveno, L. Moseley \\ University of Texas Health Science Center at Houston - D. Soebbing-Cross, J. Martinez, B. Glenn-Cole, L. Gilstrap \\ Wake Forest University Health Sciences - P. Meis, M. Swain, K. Johnson, K. Lanier, C. Leftwich \\ The George Washington University Biostatistics Center - E. Thom, Y. Zhao, V. Momirova, A. Braga, E. Cardenas, L. Leuchtenburg \\ Eunice Kennedy Shriver National Institute of Child Health and Human Development - S.Tolivaisa \\ MFMU Network Steering Committee Chair (University of Texas Medical Center, Galveston, TX) - G. Anderson, MD
}


${ }^{9}$ The Ohio State University, Columbus, $\mathrm{OH}$

${ }^{10}$ Case Western Reserve University, Cleveland, $\mathrm{OH}$

${ }^{11}$ University of North Carolina, Chapel Hill, NC

${ }^{12}$ Wayne State University, Detroit, MI

${ }^{13}$ Brown University, Providence, RI

${ }^{14}$ University of Texas Southwestern Medical Center, Dallas, TX

${ }^{15}$ University of Texas Health Science Center at Houston, Houston, TX

${ }^{16}$ Wake Forest University Health Sciences, Winston-Salem, NC

\section{Abstract}

Objective-To define the pharmacokinetic (PK) parameters of 17-hydroxyprogesterone caproate (17-OHPC) in multifetal gestation.

Study Design-Blood was obtained at 24-28 weeks and at 32-35 weeks in 97 women with twin and 26 women with triplet gestation receiving 17-OHPC. Six of the women with twins had daily blood sampling for 7 days between 24 and 28 weeks and PK parameters were estimated using noncompartmental analysis. Modeling was applied to estimate the population parameters and to simulate various treatment scenarios.

Results-The apparent half-life of 17-OHPC was 10 days. BMI significantly impacted 17OHPC concentrations but fetal number and parity did not. Apparent clearance was significantly greater in African American than in Caucasian women ( $\mathrm{p}=0.025)$.

Conclusions-This is the first pharmacokinetic analysis of 17-OHPC in pregnant women. Determination of half life, covariates affecting plasma 17-OHPC concentrations and modeling of drug behavior provide insights into this drug's pharmacology during multifetal pregnancy.

\section{Keywords}

17-hydroxyprogesterone caproate; pharmacokinetics; multifetal pregnancy

\section{Introduction}

17-hydroxyprogesterone caproate (17-OHPC) reduces the rate of recurrent preterm birth in women carrying a single fetus. This therapy has been evaluated in other conditions associated with preterm birth including multifetal gestation, $, 2,3,4$ short cervix,, 5 and cervical cerclage. ${ }^{7}$, Despite widespread clinical use, no data exist describing the pharmacokinetics of 17-OHPC in pregnancy or the plasma concentrations achieved during therapy for preterm birth prevention. In the current study, we evaluated the pharmacokinetics (PK) of 17-OHPC in women with either a twin or triplet gestation who were receiving 17-OHPC in one of two separate placebo controlled trials aimed at determining the utility of this agent in reducing preterm birth. ${ }^{2,3} \mathrm{We}$ also used population pharmacokinetic modeling to simulate plasma 17OHPC concentrations under various clinical conditions.

\section{MATERIALS AND METHODS}

\section{Patients and Drug administration}

A total of 661 women with twins and 134 women with triplet gestation were recruited into two randomized controlled trials. ${ }^{2,3}$ Subjects received weekly injections of either $250 \mathrm{mg}$ $17-\mathrm{OHPC}$ in $1 \mathrm{ml}$ castor oil or $1 \mathrm{ml}$ castor oil alone from the time of enrollment (16 0/7 
weeks - 20 6/7 weeks) until 35 weeks unless delivered earlier. Data recorded for each patient included maternal age, parity, race, BMI and gestational age at enrollment, as well as gestational age at each blood sampling and at delivery. These data were evaluated as covariates in the pharmacokinetic analysis. This study was approved by the institutional review boards of each clinical site and of the data coordinating center. Consent was given before enrollment into the study. The parent trials were registered at Clinical Trials.gov (NCT00099164). ${ }^{2,3}$

\section{Pharmacokinetic sampling schedule}

Among subjects recruited for the primary randomized controlled trials and receiving all their scheduled injections of 17-OHPC, 97 with twins and 53 with triplets were undelivered and had a single blood sample drawn between 24-28 weeks (epoch1) for measurement of 17OHPC concentration. Among these women, 70 with twins and 26 with triplets were undelivered and had a second sample taken at 32-35 weeks (epoch 2) for analysis. The infrequent (sparse) sampling described above is useful in comparing plasma 17-OHPC concentrations over time and between groups but does not lend itself to classic pharmacokinetic analysis which requires frequent sampling during one dosing interval. Fifteen of the 97 women with twins agreed to have a single blood sample taken daily for seven consecutive days over a dosing interval of one week between 24-28 weeks (intensive sampling). The first blood sample was drawn minutes prior to a scheduled injection. Recruitment of these 15 women was masked to treatment arm; therefore women who received either 17-OHPC or placebo were included. All of the fifteen women had received a minimum of four weekly injections of 17-OHPC from the time of enrollment in anticipation that steady state concentration in those women receiving 17-OHPC would be achieved by the start of the PK study. Analysis of these plasma samples was not undertaken until completion of the clinical trials so that masking of treatment arm was maintained

\section{Sample Analysis}

For all 17-OHPC measurements, blood was collected in $10 \mathrm{ml}$ tubes with EDTA as the anticoagulant and centrifuged within one hour at $3500 \times \mathrm{g}$ for 10 minutes. The supernatant plasma was aliquoted to $1 \mathrm{ml}$ tubes and frozen at -70 degrees centigrade until analyzed by high performance liquid chromatography with tandem mass spectrometry (LC-MS). The assay methodology has been reported. ${ }^{8}$ The lower limit of detection of the assay for 17OHPC was $1 \mathrm{ng} / \mathrm{ml}$, inter and intra assay variability at $10 \mathrm{ng} / \mathrm{ml}$ were 7.9 and $5.2 \%$, respectively. The analyst and the clinical centers involved in recruitment remained masked to the treatment assignment until the analyses were completed.

\section{Noncompartmental Pharmacokinetic Analysis}

Nine of the 15 subjects who underwent "intensive sampling" (daily for seven days) had received placebo and were therefore not included in the pharmacokinetic analysis though their plasma samples had been analyzed. Pharmacokinetic parameters for the 6 subjects who received 17-OHPC and underwent "intensive sampling" were estimated using the standard noncompartmental approach implemented in WinNonlin ${ }^{\circledR}$ (v. 4.0, Pharsight Corp., Mountain View, CA). Trough concentrations (Ctrough) maximum concentration (Cmax) and time to maximum concentration (Tmax) were determined from the observed data. The elimination rate constant $(\lambda z)$ was determined by log-linear regression of the terminal linear disposition phase. Half-life (t1/2) was estimated by $0.693 / \lambda z$. Area under curve (AUC) was calculated using the linear trapezoidal method. Apparent clearance [clearance (CL)/ bioavailability (F)] was estimated by Dose/(AUC), and the apparent volume of distribution (VD/F) was calculated by Dose/( $\lambda z$ AUCinf). 


\section{Population Pharmacokinetic Analysis}

Population pharmacokinetics is the pharmacokinetic evaluation of patients who are representative of the target patient population being treated with a medication. This methodology is commonly used to define patient specific factors such as weight, race or sex that contribute to the variability in drug concentrations. Large populations of subjects with fewer samples taken from each subject can be analyzed with this methodology. With a sufficiently large population, estimates of the impact of covariates on pharmacokinetic parameters can be made without having to collect numerous samples from each subject. For the current study, analysis was performed by means of non-linear mixed effects modeling implemented in the Monolix ${ }^{\circledR}$ software. Both sparsely sampled and intensively sampled data used in the non-compartmental analysis were included in the dataset.

Preliminary analysis for the structural model was performed by comparing one-compartment model with two and three compartment models. A proportional error model was used for describing residual variability. Interindividual variability was assumed to have a log-normal distribution. The patient characteristics BMI, race, fetal number, and parity were evaluated as covariates during the model building process as described by Lavielle and Mentre. ${ }^{9}$ The goodness of fit of the final model was evaluated by inspecting the following charts: scatterplots of predictions (population and individual) versus individual observations; population weighted residuals versus predictions and independent variable (time); absolute individual weighted residuals versus individual predictions. In addition, model validation was performed using prediction distribution errors and visual predictive check, obtained by conducting 1000 Monte Carlo simulations of the data set for the final model. ${ }^{9,10}$

Further, simulations were carried out based on the final model to evaluate the effect of changes in BMI and dosing regimen on the plasma concentration time profiles of 17-OHPC.

\section{Statistical Analysis}

GraphPad Prism (4.01) was used for performing the statistical tests for significance. Nonparametric (Mann Whitney U) tests were used for group comparisons. Kruskal-Wallis test with Dunn's multiple comparison was used for testing equality of population medians in multiple groups. We considered p-values $<0.05$ to be significant.

\section{RESULTS}

\section{Pharmacokinetics (Intensive Sampling)}

As expected, among the women who agreed to intensive (daily) sampling, 17-OHPC was not detectable in the plasma of the 9 women who received placebo but was detectable in all six women who had received 17-OHPC. Figure 1 depicts the mean $( \pm \mathrm{SD})$ plasma concentration of 17-OHPC in the six women with twins who had daily blood sampling over a 7 day period. Average peak concentration (Cmax) of $17.3 \mathrm{ng} / \mathrm{ml}$ was noted at the first post injection sample at 1 day. Over the ensuing 6 days, plasma concentrations declined slowly to a nadir of $9.7 \mathrm{ng} / \mathrm{ml}$ at 7 days post injection. Selected pharmacokinetic parameters (mean, SD and range) for 17-OHPC in these 6 subjects are summarized in Table 1. The apparent half life was long at 10 days suggesting slow release from either the castor oil depots or maternal fat. The wide range of AUC and Cmax values indicates considerable interindividual variation in absorption and metabolism of 17-OHPC. The peak concentration (Cmax) occurred 1.2 days (Tmax) after the injection. The apparent volume of distribution was large with considerable inter-individual variation. Apparent clearance varied two-fold with a mean of 1204 1/day. 


\section{Steady State Concentration}

We also evaluated whether steady state concentrations of 17-OHPC were achieved over the course of therapy (Figure 2). For this analysis only women who received all their scheduled injections and remained undelivered through epoch 2 (32-35 weeks) were included. This included 70 women with twins and 12 women with triplets. Among these women, the mean plasma concentrations of 17-OHPC were higher in epoch 2 than in epoch 1 for both the twin and triplet groups and significantly so in the twin group $(\mathrm{p}=0.002)$. The mean time from first injection to first blood draw was 43.2 (sd 12.5) days for twins and 45.0 (sd 12.0) days for triplets. The mean time between first and second blood draw was 46.1 (sd 8.2) days for twins and 44.3 (sd 5.8) days in women with triplets. Since plasma concentrations at epoch 2 (32-35 weeks) were higher than at epoch 1 (24-28 weeks) steady state concentrations were not achieved by epoch 1 .

\section{Impact of BMI and other covariates on 17-OHPC concentrations}

We evaluated the impact of pre-pregnancy BMI, race and parity on plasma 17-OHPC concentrations only in women with twins. In this cohort we evaluated separately the 97 women who had received all their scheduled injections of 17-OHPC and remained undelivered until the first blood sample was drawn between 24-28 weeks gestation (epoch 1) and the 70 women who received all their scheduled injections and remained undelivered at the second blood draw at $32-35$ weeks (epoch 2). A significant, $(\mathrm{p}<0.01)$ albeit weak linear relationship ( $\mathrm{r}=-0.28$ for epoch 1 and -0.33 for epoch 2 ) was observed for plasma 17OHPC and BMI in women with twins (data not shown). Plasma concentrations of 17-OHPC in African Americans (AA) in epoch 2 only were lower $(\mathrm{p}=0.051)$ compared with Caucasians (CA). Parity did not impact plasma 17-OHPC concentrations significantly in either epoch.

\section{Impact of Fetal Number of 17-OHPC Concentration}

Concentrations of 17-OHPC (mean and median) did not differ significantly in women carrying triplets compared with women carrying twins. This applied to both sample time points (Figure 2).

\section{Population Pharmacokinetics (POP-PK)}

A total of seventy-one patients with twin gestation (65 in the sparsely sampled group and 6 in the intensively sampled group) who had at least two samples drawn during the study were included in the POP-PK model building process. A total of 188 observational data points were collected and utilized for this analysis. The data was best described by a onecompartment model with first order absorption. Of the various covariates tested in building the final model, BMI was observed to have a significant effect on the estimation of 17OHPC clearance and was included in the final model. Although race was not a significant covariate in the final model, plasma clearance in African Americans was significantly higher than in Caucasians $(\mathrm{p}<0.05)$ (Fig.3).

The estimates of the pharmacokinetic parameters for these 71 subjects and their respective standard errors are shown in Table 2. The inter-individual variability (IIV) was observed to be $\sim 24 \%$ for clearance and $\sim 49 \%$ for volume of distribution. The correlation coefficients between predicted vs observed concentrations of 17-OHPC for individual and population estimates were 0.85 and 0.50 , respectively. This indicates the model fit to be fairly good for each individual subject data. The observed variability in plasma concentrations between subjects was reduced when adjusted for covariates, but other variables not collected in the study also could have contributed to the additional variability in plasma concentrations. 


\section{Plasma Concentration Simulations}

We utilized pharmacokinetic parameters obtained by POP PK analysis to simulate plasma 17-OHPC concentrations under various clinical scenarios. Simulations were carried out to explore the effect of changes in BMI and dosing regimen on the plasma concentration time profiles of 17-OHPC (Figure 4). The plasma concentration of 17-OHPC varied significantly with BMI (Figure 4a) showing a more than two-fold difference in concentration over the BMI range seen in our subjects $\left(18-45 \mathrm{~kg} / \mathrm{m}^{2}\right)$.

The impact of a loading dose on time needed to reach steady state concentrations was also evaluated. A simulated loading dose of $1000 \mathrm{mg}$ reached and maintained steady state within one week (Figure $4 \mathrm{~b}$ ). The final simulated steady state concentrations achieved with a loading dose of $1000 \mathrm{mg}$ followed by weekly injections of $250 \mathrm{mg}$ would be comparable to those observed without a loading dose. We also evaluated the effect of changing the dosing schedule on the concentration-time profile (Figure 4c). Administering a dose of $500 \mathrm{mg}$ once every 2 weeks achieved predicted steady state concentrations similar to those with a $250 \mathrm{mg}$ weekly injection although peaks were about $15 \%$ higher and trough concentrations were about $15 \%$ lower with the $500 \mathrm{mg}$ dose.

\section{Comment}

This is the first report of plasma concentrations and pharmacokinetic analysis of 17-OHPC in pregnant women. ${ }^{11}$ Only one other study has evaluated the pharmacokinetics of 17 OHPC but that was in non-pregnant women. ${ }^{12}$ Pharmacokinetic evaluation has not been reported in pregnant women with singleton gestation receiving 17-OHPC, so this study in women with multifetal gestations provides an opportunity to evaluate this medication in pregnancy. Our data provide insight into the general pharmacological properties of this agent. We have shown that the half-life of 17-OHPC is long, that plasma concentrations are affected by maternal BMI but not by fetal number or parity. We also demonstrated higher clearance and lower concentrations of the drug in African American compared with Caucasian women. We have simulated plasma 17-OHPC concentrations and have demonstrated how maternal BMI would affect plasma concentrations and how alternative dosing regimens would affect targeted plasma concentrations.

The half life of 17-OHPC in twins was long at 10 days. This long half life coupled with our demonstration that 17-OPHC is rapidly metabolized in vitro by human hepatocytes and liver microsomes ${ }^{13}$ suggests that slow release from the castor oil depot or maternal body fat determines terminal half-life of 17-OHPC rather than the drug's metabolism or elimination characteristics. A long half life is also seen in non-pregnant women and can be expected in pregnant women with singleton gestation.

The time to achieve peak concentration of 17-OHPC was 3-7 days in non-pregnant women ${ }^{12}$, whereas we noted peak concentrations at 1-2 days after an injection. We did not sample prior to the 24 hours time point so it is possible that in women with twins, the peak plasma concentrations after an intramuscular injection occurs before 24 hours in women carrying twins. Nonetheless, it appears that the time to peak concentration after an intramuscular injection is shorter in pregnant women than in non-pregnant women. This finding is not unexpected as blood flow to most tissues in pregnancy is increased compared with that in non-pregnant subjects. The rate of rise in plasma 17-OHPC concentrations is slow compared with intramuscular injections of other drugs dissolved in water based solvents. ${ }^{14}$ The castor oil solvent slowly releases the 17-OHPC. The time to rapidly achieve the desired concentration would become more relevant if other indications requiring a rapid onset of action of 17-OHPC are evaluated in clinical trials (eg treatment of preterm labor). 
We selected to obtain blood samples at two time points. The first at 24-28 weeks was selected in anticipation that steady state would have been reached since women began their injections between 16-20 weeks gestation. The second sample was obtained at 32-35 weeks to evaluate whether the drug accumulated with repeated injections. Steady state concentration however, was not achieved by the time of the first blood draw. The reason steady state is not achieved is likely due to the continuous, slow release of 17-OHPC from the castor oil depots which is augmented with each injection. The therapeutic concentration for 17-OHPC has not been established, but once it is, a loading dose could be administered if therapeutic concentrations need to be rapidly achieved.

In this study, the pharmacokinetic parameters calculated from compartmental analysis were used to simulate 17-OHPC plasma concentration time profiles under multiple hypothetical clinical scenarios. The simulations were based on pharmacokinetic parameters estimated from population pharmacokinetic analysis and included all the samples (trough concentrations and PK samples) and so are not constrained by the potential error in any half life estimates based on the pharmacokinetic study. The simulations we performed from women with twin gestation are useful in providing a perspective of the drug's behavior in pregnancy. With modeling, we were able to demonstrate a significant impact of BMI and race on plasma 17-OHPC concentrations. The basis for these differences is unclear but the racial differences in 17-OHPC clearance suggest the possibility that genetic factors may influence 17-OHPC pharmacologic behavior. Once therapeutic concentrations of 17-OHPC are determined, higher doses may be needed to achieve desired concentrations in obese women and in African-American women to achieve desired concentrations.

Simulations were also conducted to determine other possible dosing strategies. For example, we demonstrated that a dosing schedule of $500 \mathrm{mg}$ administered once every two weeks could be utilized to achieve plasma concentrations comparable to those achieved with the currently utilized regimen of $250 \mathrm{mg}$ weekly. This regimen would clearly improve patient's acceptability. However, this dosing schedule needs to be evaluated carefully since the peak/ trough fluctuations were greater in this case as compared to the $250 \mathrm{mg} / \mathrm{week}$ regimen. Furthermore, these simulation data cannot be applied to women with a singleton gestation. We also demonstrated with our simulations that, if there was clinical need to achieve target steady state concentrations rapidly, this could be done with a loading dose. The issue of safety of such an approach would require consideration.

Conclusions regarding 17-OHPC pharmacokinetics reached in women with multifetal gestations may not be applicable to women with singleton gestations. However, the findings in women with a twin or triplet gestation provide a basis for evaluating the impact of covariates on the pharmacology of 17-OHPC in singleton gestations. Similar simulations with ritodrine proved useful in defining the association between drug dose and side effects and in demonstrating the harmful effects of rapid dose escalation. ${ }^{15}$

17-hydroxyprogesterone caproate is recommended as a treatment option for women with a prior preterm birth. ${ }^{16}$ Vaginal progestins have not proven consistently effective in this population ${ }^{17}$ so 17-OHPC continues to be the therapeutic option of proven benefit for this indication. ${ }^{1}$ Despite the widespread use of this agent, very little pharmacologic information exists as to the proper dosing regimen or the mechanism of action of the drug. ${ }^{18,19,20,21,22,23}$ The currently utilized regimen of $250 \mathrm{mg}$ injected intramuscularly weekly is empiric. Our study indicates a wide inter-individual variation in the pharmacology of 17-OHPC. Such wide variability suggests that the beneficial effect of 17-OHPC in reducing preterm birth rates in singleton gestation may be further optimized with a drug administration regimen that achieves the desired concentration. 
In conclusion, this is the first report of plasma concentrations of 17-hydroxyprogesterone caproate in pregnant women. This report defines the pharmacokinetic behavior of 17hydroxyprogesterone caproate in women with twin gestation and the impact of BMI, race and fetal number on plasma 17-OHPC concentrations. Whether substantive differences in these observations will be seen in singleton gestation requires additional study.

\section{Acknowledgments}

The project described was supported by grants from the Eunice Kennedy Shriver National Institute of Child Health and Human Development (HD27869, HD21410, HD40512, HD34136, HD34208, HD40485, HD27915, HD40544, HD40560, HD27917, HD40500, HD34116, HD40545, HD27860, HD36801) and does not necessarily represent the official views of the NICHD or the National Institutes of Health.

The authors wish to thank the following Network members for their contributions: Elizabeth Thom, PhD, Yuan Zhao, MS and Valerija Momirova, MS for protocol/data management and statistical analysis; and Margaret Cotroneo, RN and Allison Northen, RN, BSN for protocol development and coordination between clinical research centers.

\section{References}

1. Meis, et al. for the NICHD Maternal-Fetal Medicine Network. Prevention of recurrent preterm delivery by 17 alpha-hydroxyprogesterone caproate. N Engl J Med. 2003; 348:2379-2385. [PubMed: 12802023]

2. Rouse DJ, et al. for the NICHD Maternal-Fetal Medicine Units Network. A trial of 17 alphahydroxyprogesterone caproate to prevent prematurity in twins. N. Engl J Med. 2007; 357:454-461. [PubMed: 17671253]

3. Caritis SN, et al. for the NICHD Maternal-Fetal Medicine Units Network. Prevention of preterm birth in triplets using 17 alpha-hydroxyprogesterone caproate a randomized controlled trial. Obstet \& Gynecol. 2009; 113:285-292.

4. Combs CA, Carite T, Maurel K, Das A, Porto M. Obstetrix Collaborative Network. Failure of 17hydroxyprogesterone to reduce neonatal morbidity or prolong triplet pregnancy: a double-blind, randomized clinical trial. Am J Obstet Gynecol. 2010 Sep; 203(3):248.e1-248.e9. [PubMed: 20816146]

5. Berghella V, Figueroa D, Szychowski JM, Owen J, Hankins GD, Iams JD, Sheffield JS, PerezDelboy A, Wing DA, Guzman ER. Vaginal Ultrasound Trial Consortium. 17-alphahydroxyprogesterone caproate for the prevention of preterm birth in women with prior preterm birth and a short cervical length. Am J Obstet Gynecol. 2010 Apr; 202(4):351.e1-351.e6. [PubMed: 20350641]

6. Keeler, Sm; Kiefer, D.; Rochon, M.; Quinones, JN.; Novetsky, AP.; Rust, O. A randomized trial of cerclage vs. 17-alpha-hydroxyprogesterone caproate for treatment of short cervix. J of Perinat Med. 2009; 37(5):473-479. [PubMed: 19492920]

7. Rebarber A, Cleary-Goldman J, Istwan NB, Rhea DJ, Desch C, Russo-Stieglitz K, Saltzman DH. The use of 17 alpha-hydroxyprogesterone caproate (17P) in women with cervical cerclage. Am J Perinatol. 2008; 25:271-275. [PubMed: 18401840]

8. Zhang S, Mada SR, Torch M, Mattison D, Caritis SN, Venkataramanan R. Simultaneous quantification of 17 alpha-hydroxyprogesterone caproate, 17 alpha-hydroxyprogesterone and progesterone in human plasma using high performance liquid chromatography-mass spectrometry (HPLC-MS/MS). J Pharm Biomed Anal. 2008 Dec 1; 48(4):1174-1180. Epub 2008 Aug 29. [PubMed: 18947956]

9. Lavielle M, Mentre F. Estimation of population pharmacokinetic parameters of saquinavir in HIV patients with the MONOLIX software. J Pharmacokinet Pharmacodyn. 2007; 34:229-249. [PubMed: 17211713]

10. Bonate PL. A brief introduction to Monte Carlo simulation. Clin Pharmacokinet. 2001; 40:15-22. [PubMed: 11236807] 
11. Pubmed, June 1954- June 3, 2009, English language journals, keywords: delalutin, tocolytic, hexanoate, 17-P, 17-OHPC, 17 hydroxyprogesterone caproate, 17 hydroxyprogesterone hexanoate, drug concentrations, pharmacokinetics

12. Onsrud M, Paus E, Huag E, Kjorstad K. Intramuscular administration of hydroxyprogesterone caproate in patients with endometrial carcinoma. Acta Obstet Gynecol Scand. 1985; 64:519-523. [PubMed: 2932883]

13. Sharma S, Ou J, Strom S, Mattison D, Caritis SN, Venkataramanan R. for the NICHD ObstetricFetal Pharmacology Research Units (OPRU) Network. Identification of Enzymes involved in the metabolism of $17 \alpha$-hydroxyprogesterone caproate: an effective agent for prevention of preterm birth. Drug Metabolism and Disposition. 2008; 36:1896-1902. [PubMed: 18573861]

14. Caritis SN, Venkataramanan R, Cotroneo M, Smith M, Chiao JP, Habucky K. Pharmacokinetics and pharmacodynamics of ritodrine after intramuscular administration of pregnant women. Am J Obstet Gynecol. 1990; 162(5):1215-1219. [PubMed: 2339723]

15. Caritis SN, Venkataramanan R, Darby MJ, Chiao JP, Krew M. Pharmacokinetics of ritodrine administered intravenously: recommendations for changes in the current regimen. Am J Obstet Gynecol. 1990 Feb; 162(2):429-437. [PubMed: 2309826]

16. ACOG Committee Opinion. Vol. 419. 2008 October.

17. O’Brien JM, Adair CD, Lewis DF, Hall DR, DeFranco EA, Fusey S, Soma-Pillay P, Porter K, How H, Schackis R, Eller D, Trivedi Y, VanBuren G, Khandelwal M, Trofatter K, Vidyadhari D, Vijayaraghavan J, Weeks J, Dattel B, Newton E, Chazotte C, Valenzuela G, Calda P, Bsharat M, Creasy GW. Progesterone vaginal gel for the reduction of recurrent preterm birth: primary results from a randomized, double-blind, placebo-controlled trial. Ultrasound Obstet Gynecol. 2007; 30:687-696. [PubMed: 17899572]

18. Attardi BJ, Zeleznik A, Simhan H, Chiao JP, Mattison DR, Caritis SN. Obstetric-Fetal Pharmacology Research Unit Network. Comparison of progesterone and glucocorticoid receptor binding and stimulation of gene expression by progesterone, 17-alpha hydroxyprogesterone caproate, and related progestins. Am J Obstet Gynecol. 2007 Dec; 197(6):599.e1-599.e7. [PubMed: 18060946]

19. Foglia LM, Ippolitio DL, Stallings JD, Zelig CM, Napolitano PG. Intramuscular 17hydroxyprogesterone caproate administration attenuates immunoresponsiveness of maternal peripheral blood mononuclear cells. Am J Obstet Gynecol. 2010 Dec; 203(6):561.e1-561.e5. [PubMed: 20810098]

20. Patel S, Li A, Goodwin TM, Brower M, Blitz M, Minoo P, Felix JC, Lee RH. Effect of 17-alpha hydroxyprogesterone caproate on the production of tumor necrosis factor-alpha and the expression of cyclooxygenase-2 in lipopolysaccharide-treated gravid human myometrial explants. J Perinatol. 2010 Sep; 30(9):584-589. [PubMed: 20220763]

21. Klebanoff MA, Meis PJ, Dombrowski MP, Zhao Y, Moawad AH, Northen A, Sibai BM, Iams JD, Varner MW, Caritis SN, O’Sullivan MJ, Leveno KJ, Miodovnik M, Conway D, Wapner RJ, Carpenter M, Mercer BM, Ramin SM, Thorp JM, Peaceman AM. for the National Institute of Child Health and Human Development Maternal-Fetal Medicine Units Network. Salivary progesterone and estriol among pregnant women treated with 17-alpha-hydroxyprogesterone caproate or placebo. Am J Obstet Gynecol. 2008 Nov; 199(5):506.e1-506.e7. [PubMed: 18456237]

22. Facchinetti F, Dante G, Venturini P, Paganelli S, Volpe A. 17alpha-hydroxyprogesterone effects on cervical proinflammatory agents in women at risk for preterm delivery. Am J Perinatol. 2008 Sep.; 25(8):503-506. [PubMed: 18756431]

23. Tiboni GM, Del Corso A, Marotta F. Progestational agents prevent preterm birth induced by a nitric oxide synthesis inhibitor in the mouse. In Vivo. 2008 Jul-Aug; 22(4):447-450. [PubMed: 18712170]

\section{DEFINITIONS}

Pharmacokinetics
This term describes the time course of a drug in the body and includes absorption, distribution, metabolism and elimination. 


\section{Pharmacodynamics}

Sparse sampling

Intensive sampling

One compartment model

Multi compartment model

Steady state
concentration

C max

Tmax or T peak

Area under the concentration -time curve

Clearance

Volume of distribution
In simple terms it is what the body does to an administered drug.

This describes the relationship between the pharmacological response and the drug concentration. This includes physiologic or biochemical effects of drugs on the body or on microorganisms or parasites within or on the body.

A technique by which a limited number of samples is obtained from a large population of subjects taking the same medication in order to make inferences about the drug or its response.

This is typically done within a dosing interval in order to characterize the pharmacokinetics of a drug in a given patient

If a drug distributes instantaneously through out the entire body it is said to exhibit a one compartment model.

If a drug does not distribute instantaneously through out the entire body it is said to exhibit a multi compartment model. Under such a condition while the drug distributes instantaneously to certain parts of the body, it takes a longer time to reach other parts of the body.

Steady state concentrations of a drug are achieved when the rate at which a drug comes into the body equals the rate at which the drug leaves the body. At steady state, the plasma concentration of a drug is constant during continuous intravenous infusion, or the plasma concentration versus time profile during a dosing interval is identical to the plasma concentration versus time profile during the subsequent dosing intervals for a fixed dose and dosing frequency. Steady state plasma concentrations are achieved in approximately 5-6 half lives.

This is the highest concentration of a drug in blood or plasma during a dosing interval after administration of a drug.

This is the time at which maximum blood or plasma concentrations are achieved during a dosing interval.

This is the area under the blood or plasma concentration versus time curve for a drug.

This describes the over all ability of the body to clear the drug. It is the volume of blood or plasma that is completely cleared of the drug per unit time. The clearance is calculated as the amount of drug cleared (for IV dose this will be dose) divided by the area under the blood or plasma concentration versus time.

Volume of distribution is a hypothetical volume that relates the concentration of the drug in the measured biological fluid (normally plasma or serum or blood) to the amount of drug in the body. In other word, it is the apparent volume into which the drug has to be distributed at a concentration equal to the 


\author{
Apparent Half-life \\ Simulation of plasma
concentrations
}

Monte Carlo

simulations

Population

pharmacokinetics

(POP - PK) concentration measured in the biological fluid. It is typically expressed in liters or in liters per kilogram. This parameter provides information about the extent to which the drug is distributed outside the vascular system.

This is a measure of the time that it takes for the drug concentration to decrease from a given value to one half of its value. It takes about 5-6 half lives for most of the drug to be out of the body. It takes about 5-6 half lives to reach steady state.

This is an approach that utilizes the pharmacokinetic parameters of a drug and mathematical modeling to predict the blood or plasma concentration versus time for various doses and dosing frequencies.

A problem solving technique used to approximate the probability of certain outcomes by running multiple trial runs, called simulations, using random variables.

This describes the relationship between physiology and pharmacokinetics and pharmacodynamics. Population pharmacokinetics is the study of the sources and correlates of variability in drug concentrations in the patient population receiving clinically relevant doses of a drug of interest. 


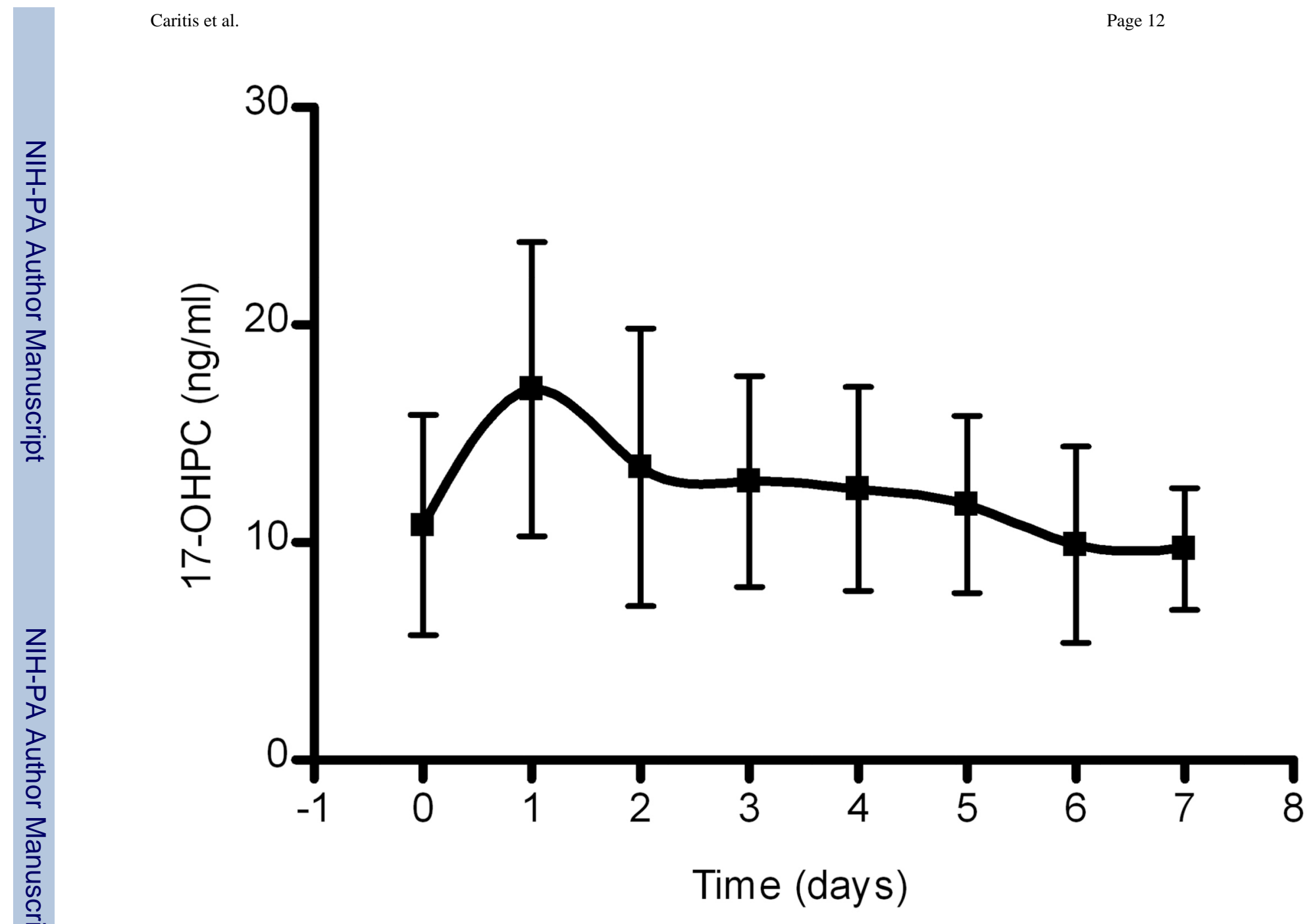

Figure 1. Mean Plasma Concentration of 17-OHPC Following IM Injection of 250mg 17-OHPC concentration-time profiles for 6 subjects with twins who had sampling done before and then daily for seven days after an injection. Values are mean $( \pm)$ standard deviation. 


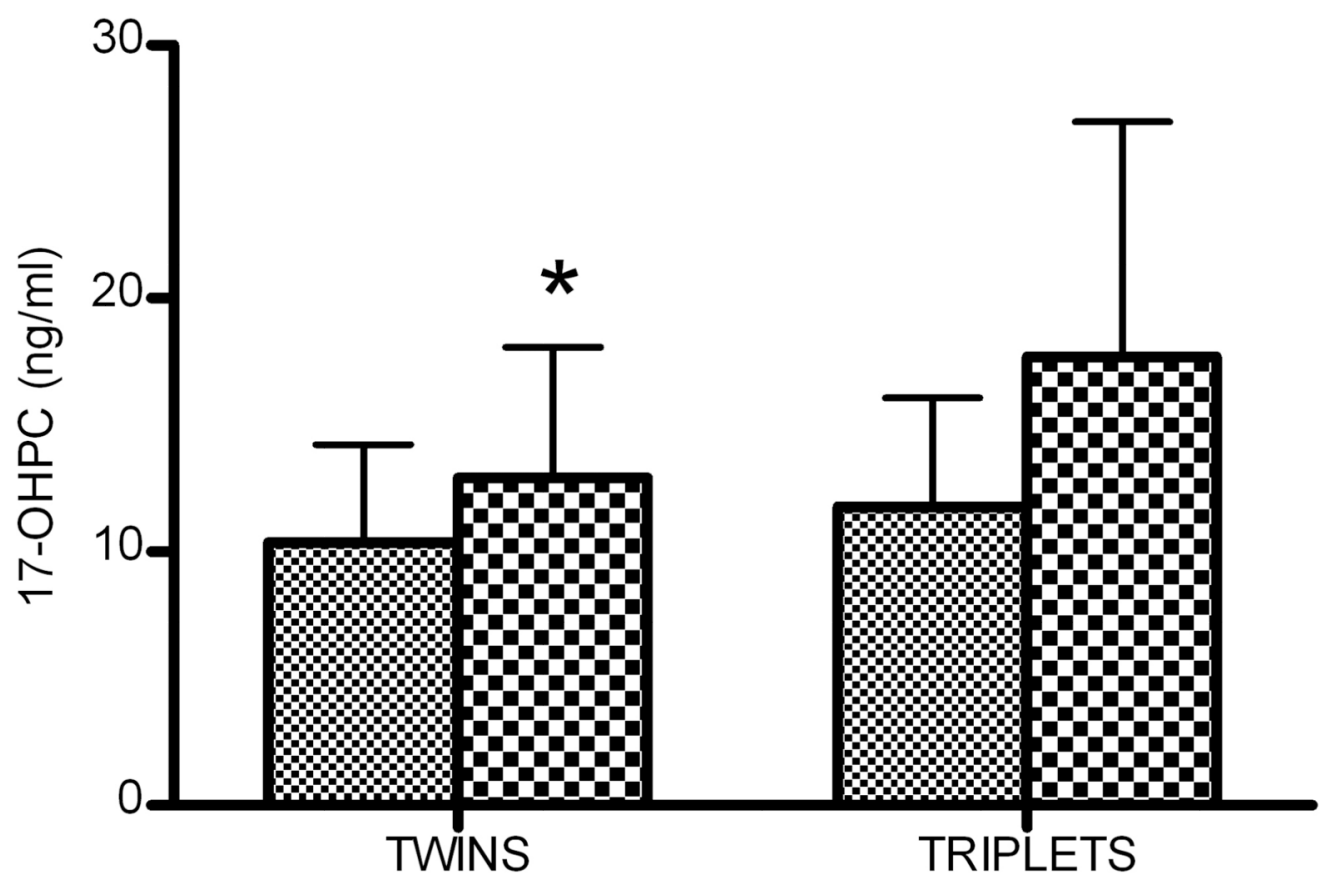

$\infty \infty \times$ EPOCH 1

EPOCH 2

Figure 2. Mean Plasma Concentrations of 17-OHPC in Twin and Triplet Gestation During Two Gestational Epochs

Mean ( \pm sd) 17-OHPC concentrations in the 70 subjects with twins and the 12 subjects with triplets who had blood obtained during epoch 1 at 24-28 weeks and epoch 2 at 32-35 weeks. Single asterisk indicates significant difference $(\mathrm{p}<0.002)$ between epoch1 and epoch 2 in twins. Concentrations in twins vs triplets were statistically similar $(\mathrm{p}>0.05)$ both in epoch 1 and 2. Mann-Whitney test was used for the comparisons. 


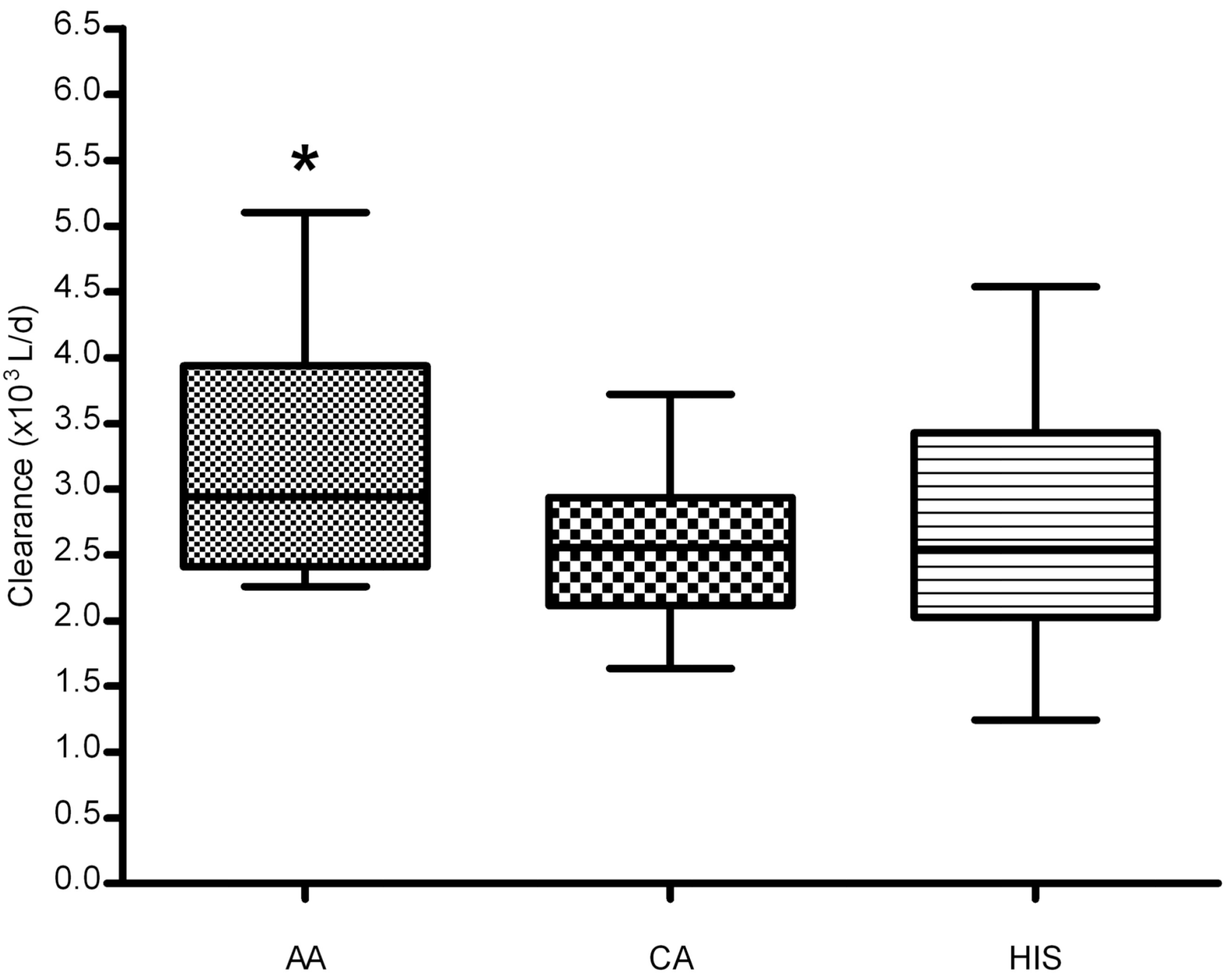

Figure 3. 17-OHPC Clearance According to Race

A boxplot depicting the median 17-OHPC clearance (individual) estimates associated with different ethnicities. The bars represent $25^{\text {th }}$ (lower bar) and $75^{\text {th }}$ (upper bar) percentile. AA - African Americans $(n=14), C A-$ Caucasians $(n=46)$ and HIS - Hispanics $(n=9)$. The mean clearance in the Caucasians was significantly lower than that of African Americans $(\mathrm{p}<0.05)$. The individual estimates were obtained with the final model which included BMI as the covariate. Kruskal-Wallis one-way analysis of variance with Dunn's post test was used for the comparison. 

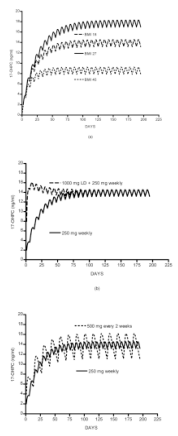

Figure 4. Simulated Plasma 17-OHPC Concentrations

Simulated plasma concentration time profiles of 17-OHPC in pregnant subjects. The bold indicates the simulated plasma concentration utilizing a dose of $250 \mathrm{mg}$ IM once weekly of 17-OHPC. The lighter line indicates simulated concentrations under various scenarios.

a. effect of BMI $(18,45$ and 27) on plasma concentration time profiles,

b. effect of adding a loading dose of $1000 \mathrm{mg}$ to the currently recommended regimen, and

c. effect of changing the dosing schedule from $250 \mathrm{mg}$ once weekly to $500 \mathrm{mg}$ every 2 weeks. 


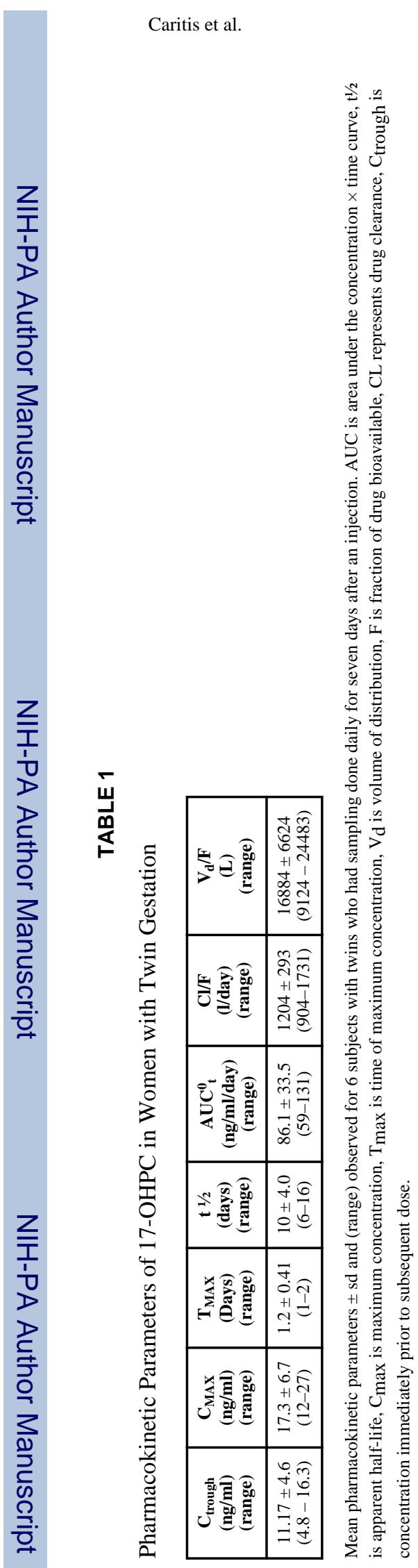

Am J Obstet Gynecol. Author manuscript; available in PMC 2012 July 1. 
TABLE 2

Population Pharmacokinetic Parameter Estimates

\begin{tabular}{|c|c|}
\hline Parameter & Mean \pm SE \\
\hline Ka $\left(\mathbf{d a y}^{-1}\right)$ & 1.5 (Fixed) \\
\hline V/F $\left(\times \mathbf{1 0}^{\mathbf{3}}\right.$ ltr $)$ & $62.5 \pm 7.2$ \\
\hline Cl/F $\left(\times \mathbf{1 0}^{\mathbf{3}} \mathbf{l t r} / \mathbf{d}\right)$ & $1.29 \pm 0.24$ \\
\hline IIV_V & $0.49 \pm 0.13$ \\
\hline IIV_Cl & $0.24 \pm 0.04$ \\
\hline
\end{tabular}

Population pharmacokinetic parameter estimates (mean \pm SE) obtained from the final model. Ka: absorption rate constant, V/F: volume of distribution/bioavailability, CL/F: apparent clearance/bioavailability, IIV: Interindividual variability in estimated pharmacokinetic parameters. 